\title{
ReviewArticle
}

\section{TO WHAT EXTENT DOES EXPOSURE TO PRENATAL STRESS AFFECT OFFSPRING BEHAVIOURALOUTCOMES}

\author{
Areej-Noor Yousaf Shah
}

\section{Medical Student}

\section{Abstract}

Background: The gestational environment influences longer-term child developmental outcomes. Various studies have been conducted to determine a correlation between prenatal stress and offspring behavioural outcomes. The objectives of this study is to review current literature relating prenatal exposure to different types of stress, including psychological (anxiety, perceived stress) and acute (adverse life events), to offspring behavioural outcomes.

Methods: Since a significant body of literature exists on this topic, it was important to systematically review evidence found using online databases such as MEDLINE and Pubmed. Articles were selected based on language, if they were published after 2002, sample size, methodology, whether or not they were in a peer-reviewed journal, and the nature of the stressor - this review considers both acute and psychological stress.

Results: More genetically informed studies should first take place before causation can be determined, but there is indubitably a relationship between prenatal stress and negative child behavioural outcomes.

Conclusion: There is ample evidence of an association between maternal stress during gestation and negative offspring behavioural outcomes although a clear causative relationship cannot be established. The mechanism by which psychological stress affects behavioural outcomes are not fully known and more research could be done in future.

Keywords: Stress, psychological stress, anxiety, maternal, child behaviour 


\section{Introduction}

It is acknowledged in the "foetal origins hypothesis" (2) that the gestational environment influences longer-term child developmental outcomes. While Barker's original hypothesis focuses primarily on incidence of coronary heart disease, the mechanism by which certain maternal hormones can move transplacentally and enter the amniotic fluid (bathing the foetus at a time when it is most susceptible to its surrounding environment), and how this can cause persisting changes in a range of metabolic, physiological and structural parameters, remains the same. The impact of maternal emotional state, in particular, has been studied in recent years, (8) and there is a body of literature exploring how antenatal exposure to stressors affects child behaviour. First, however, it is necessary to define some aspects of the question. "Stress" is complex phenomenon, encompassing factors including psychological stress and stressful life events (SLEs). It has been defined as "a real or perceived perturbation to an organism's physiological homeostasis or psychological well-being (10) Behavioural outcomes" here refers mainly to child temperament, which can be assessed by surveillance or by parent-report data. In this review, autism spectrum disorder (ASD) -- a developmental disability - and attention-deficit hyperactivity disorder (ADHD), belonging to a group of behavioural symptoms including impulsiveness, inattentiveness and hyperactivity, are also identified as outcomes, and low birth weight is mentioned briefly as this is known to have consequences on child cognitive development. (6)

Various studies have been conducted to determine a correlation between prenatal stress and offspring behavioural outcomes, although it is difficult to establish causality due to a number of confounding factors. Genetics, along with the postnatal environment, can also impact the behavioural development of the child. If studies do find a causal relationship between antenatal stress and child behaviour, this can have clinical implications as it would allow preventative and therapeutic measures to be put in place for better child outcomes and mental health.

In this literature review, some higher-level studies will be discussed and analysed, which try to account for and minimise the effect of these confounding factors and covariates in order to properly determine the extent to which prenatal stress affects offspring behavioural outcomes.

Main body

\section{Psychological stress}

Psychological measures of prenatal stress were studied in a prospective longitudinal study by Huizink et al (7). Stress was divided into three predictors:

1

2.

Perced stress;

month.

All were assessed by a self-report questionnaire, which has limitations as it is not an objective measure of stress. Participants may be swayed one way or another by the way a question is worded. To measure offspring temperament, a developmental test was administered by a trained psychologist at 3 and 8 months of age. The psychologist was blind to the prenatal stress data of the mothers, making the results more valid as the possibility of bias was eradicated. After adjusting for postpartum depression and the mother's educational level, it was found that increased prenatal stress is associated with temperamental variation of young (7) Complete data was available for 170 term-born infants, so the sample size was very small, but it allowed close monitoring of a small cohort, hence more variable could be tested more easily. The article also identifies the study's limitations, most notably that it does not account for genetic influences (a confounder). In order to fully establish a causal link, however, it would be necessary to test a larger cohort.

Maternal depression and anxiety is a hugely prevalent antenatal psychological stressor often studied in conjunction with child outcomes. Based on the Avon Longitudinal Study of Parents and Children (ALSPAC), O'Connor et al. (11) (2002) conducted a larger prospective cohort study $(\mathrm{n}=7448)$ where self-report data of maternal depression and anxiety was collected at intervals throughout the pregnancy and postpartum period, followed up by parent reports on child behaviour and emotional problems at 47 and 81 months. Children whose mothers suffered from anxiety in late pregnancy demonstrated considerably more behavioural and emotional problems at 81 months when obstetric complications, postpartum depression and psychosocial disadvantage were controlled for. (11) The methodology, considering the prospective longitudinal design and the large community sample, lend this study particular power when establishing a causal link between prenatal anxiety and later child outcomes, as there will be a narrower margin of error. While Huizink et al. (7) (2002)'s study was smaller, the conclusions were similar in that a link between stress in late pregnancy and negative behavioural development was discovered. The same is true of children with VLBW (very low birth weight, $<1.5 \mathrm{~kg}$ ); (16) maternal anxiety close to labour seemed to predict child cognitive and behavioural outcomes at 24 months corrected age. Anxiety was measured using a self-report questionnaire, and the child was assessed during a video-taped playtime for internalising behavioural problems (emotionally reactive, anxious or depressed, somatic or sleep problems) and externalising problems (emotionally reactive and aggressive behaviour). Most studies of this nature exclude neonates with VLWB, so it is interesting to see similar trends between maternal anxiety and temperament. It is, however, unreasonable to assume that it is solely the influence of maternal anxiety that lead to the outcome, as low birth weight is known to cause neurodevelopmental and cognitive problems, and these are not disentangled from that of antenatal stress. Other factors may also cause VLBW including pregnancy complications and illness during pregnancy. The study cannot be dismissed completely for its quality is high. It is clear and concise, and acknowledges that maternal perinatal anxiety has effects on upbringing. Moreover, there is a degree of subjectivity during parental self-report questionnaires, so analysing child behaviour during a recorded playtime as well may give the results of this study more validity. Still, subjectivity cannot be excluded completely unless there is directly quantifiable data obtained.

A way to do this is to measure stress hormones produced.(1) did so in a prospective, longitudinal study - researchers assessed multiple indicators of maternal stress, some self-reported including anxiety, but also evaluated a physiological marker: cortisol levels, both in the maternal plasma and amniotic fluid (1). The benefit of this is it is objective, unlike the information provided in questionnaires. It also allows comparison between perceived stress and a physiologica change in the body; maternal self-reports of stress were not associated with maternal or foetal cortisol, suggesting a discrepancy exists between self-reports and physiological measurements of stress. Potentially, this dismisses other studies which do not use endocrine products as physiological markers of stress as, largely, these are unable to explain the mechanism by which it affects child behavioural outcomes. An alternative interpretation, however, is that subjective reports may be more sensitive as they come from patients themselves, so are more relevant in a clinical context. This study found that there were trend-like indirect effects of cortisol in the amniotic fluid on infant temperament via birth weight.

\section{Traumatic/ acute stress}

More acute stress and infant behaviour seem to have a similarly trendlike relationship. SLEs were assessed in one study with a yes/no questionnaire, with criteria such as "you had a serious accident or illness", "a friend/family member had a serious accident or illness" and "you were separated/divorced".(3) While these questions are straightforward and easy to answer, the retrospective measure of stressful life events may have biased the results. Furthermore, the women who were recruited for the study were predominantly white, above average age and well educated, so are not representative of a typical antenatal population. The types of women who volunteered for this study are similar to most volunteer studies taking place in a firs world country,(9) perhaps because they may have more free time not at work. Overall, results from this study indicated that SLEs in the prenatal period were negatively associated with infant mental development and behaviour. Moreover, maternal prenatal stressors including immune activation, malnutrition and adverse life events have been shown to increase risk of offspring ASD and ADHD.(15) The first trimester seemed to be a particularly vulnerable period in regards to child behavioural outcomes, as, during this time, vigorous neurogenesis occurs - a process that is disturbed by stress. This agrees with Blair et al. (4) (2011), who studied the relationship between anxiety early in gestation and child temperamental outcomes. Infan behaviour was greatly negatively influenced by higher pregnancyspecific anxiety between 13 and 17 weeks of gestation (4). A similar result was discovered in non-human primates (12). Sensitivity to prenatal stress was far greater and peaked during early gestation, and 
this tapered off in mid-late gestation. Experiments involving nonhuman primates are useful as these animals' brains share structural and functional features with humans', so any observations may be assumed to be true of humans as well, although this might not be completely accurate. Moreover, this idea that stress in the first trimester has a greater effect on child outcomes is not completely in line with other articles $(11,7)$ which focus on stress later on in the gestational period. Confounding is an issue when it comes to discussing the effect of one factor and child outcomes, and none of the studies reviewed thus far attempt to separate environmental and inherited influences, although many do account for covariates. This can alter results significantly as a confounder influences both the dependent and independent variables. Even when these factors are disentangled, similar results are obtained, proving that antenatal stress is in fact associated with adverse offspring outcomes (12). The IVF cross-fostering design employed separated inherited and environmental influences on child development. Indeed, in both unrelated and related mother-offspring pairs, associations between antenatal stress and offspring birth weight, antisocial behaviour and gestational age were seen. The implications of this is that, even when confounders are accounted for, a link is present between the two.

\section{Conclusion}

In conclusion, there is much evidence linking various types of maternal stress (including psychological stress, anxiety, perceived stress, stressful life events) during gestation to negative offspring behavioural outcomes, so it can be said with a degree of certainty that there is a relationship. From the evidence presented above, however, it is not explicitly clear whether this relationship is causative. Only one study examined uses a genetically informed design (12) so it would be unreasonable to conclude effects are causal only based on this.

Some studies stated that it was strongest when stress occurred early during gestation. $(4,15,12)$ Others found that stress later in the prenatal period played a larger role in offspring behavioural development.(11,7,16) The most vulnerable time for offspring development has therefore yet to be agreed on and established.

Unfortunately, the mechanism by which maternal antenatal stress affects behavioural outcomes of the offspring was not explained fully in this review, although many scientists accredit it to cortisol levels and the sympathoadrenal medullary and hypothalamic-pituitary-adrenal axes.(5) These, however, are all endocrine mechanisms; as of 2016, there is no known mechanism by which psychological stress affects behavioural outcomes (1). Potentially, more research could be done on this in the future.

How maternal prenatal influences can affect child outcomes so significantly is currently a relevant field of medicine, for it has implications on postnatal care and longer term child health outcomes. In this literature review, I looked mainly at the nature of the stressor, but its severity, how many stresses and if they are concentrated in a short period of time, and the point during gestation stress has the greatest influence may also affect developmental outcomes.

Overall, however, it can be agreed that exposure to prenatal stress seems to negatively affect child behaviour outcomes.

\section{References} stress, maternal plasma cortisol and cortisol in the amniotic fluid on birth outcomes and child temperament at 3 months. Psychoneuroendocrinology 38(6), pp. 907-915. doi: https://dx.doi.org/10.1016/j.psyneuen.2012.09.015

2. Barker, D. J. 1995. Fetal origins of coronary heart disease BMJ : British Medical Journal 311(6998), pp. 171-174.

3. Bergman, K. et al. 2007. Maternal Stress During Pregnancy Predicts Cognitive Ability and Fearfulness in Infancy. Journal of the American Academy of Child \& Adolescent Psychiatry 46(11), pp. 1454-1463. doi: https://doi.org/10.1097/chi.0b013e31814a62f6
4. Blair, M. M. et al. 2011. Prenatal maternal anxiety and early childhood temperament. Stress 14(6), pp. 644-651. doi: 10.3109/10253890.2011.594121

5. Entringer, S. et al. 2010. Attenuation of maternal psychophysiological stress responses and the maternal cortisol awakening response over the course of human pregnancy. Stress (Amsterdam, Netherlands) 13(3), pp. 258-268. doi: $10.3109 / 10253890903349501$

6. Huang, C et al, 2013. Cognition and behavioural development in early childhood: the role of birth weight and postnatal growth. International Journal of Epidemiology 42(1), pp. 160-171. doi: 10.1093/ije/dys207

7. Huizink, A. C. et al. 2002. Psychological measures of prenatal stress as predictors of infant temperament. Journal of the American Academy of Child and Adolescent Psychiatry 41(9), pp. 1078-1085.

8. Kinsella, M. T. and Monk, C. 2009. Impact of Maternal Stress, Depression \& Anxiety on Fetal Neurobehavioral Development. Clinical obstetrics and gynecology 52(3), pp. 425-440. doi: 10.1097/GRF.0b013e3181b52df

9. Moorcraft, S. Y. et al. 2016. Patients' willingness to participate in clinical trials and their views on aspects of cancer research: results of a prospective patient survey. Trials 17, p. 17. doi: 10.1186/s13063-015-1105-3

10. National Research Council (US) Committee on Recognition and Alleviation of Distress in Laboratory Animals. 2008. Recognition and Alleviation of Distress in Laboratory Animals. Available at: https://www.nap.edu/read/11931/chapter/4 [Accessed: 19 March 2018].

11. O'Connor, T. G. et al. 2002. Maternal antenatal anxiety and children's behavioural/emotional problems at 4 years: Report from the Avon Longitudinal Study of Parents and Children. British Journal of Psychiatry 180(6), pp. 502508. doi: $10.1192 /$ bjp. 180.6 .502

12. Rice, F. et al. 2010. The links between prenatal stress and offspring development and psychopathology: disentangling environmental and inherited influences. Psychological medicine 40(2), pp. 335-345. doi: https://dx.doi.org/10.1017/S0033291709005911

13. Schneider, M. L. et al. 1999. Growth and development following prenatal stress exposure in primates: an examination of ontogenetic vulnerability. Child development 70(2), pp. 263-274.

14. The National Institue of Mental Health. 2016. Attention Deficit Hyperactivity Disorder. Available at: https://www.nimh.nih.gov/health/topics/attention-deficithyperactivity-disorder-adhd/index.shtml [Accessed: 19 March 2018].

15. Udagawa, J. and Hino, K. 2016. Impact of Maternal Stress in Pregnancy on Brain Function of the Offspring. Nihon eiseigaku zasshi. Japanese journal of hygiene 71(3), pp. 188-194.

16. Zelkowitz, P. et al. 2011. Early maternal anxiety predicts cognitive and behavioural outcomes of VLBW children at 24?months corrected age. Acta Paediatrica 100(5), pp. 700704. doi: $10.1111 / \mathrm{j} .1651-2227.2010 .02128 . \mathrm{x}$ 